3. Keith, N. M., WAGENER, H. P. and KeRnohan, J. W. (1928).-The syndrome of malignant hypertension. Arch. Int. Med., Vol. XLI, p. 141.

4. Penfield, W. and Cone, W. (1938). - Traitement chirurgical de l'hypertension artérielle. Bull. Assoc. Med. Lang. franc. Amer. d. Nord., Vol. LXVII, p. 1154.

5. Allen, E. V. and Adson, A. W. (1938).-Physiological effects of extensive sympathectomy for essential bypertension. Further observations. Ann. Int. Med., Vol. XI, p. 2151.

6. CRAIG, W. M. and BROWN, G. E. (1934).- Unilateral and bilateral resection of the major and minor splanchnic nerves. Its effects in cases of essential hypertension. Arch. Int. Med., Vol LIV, p. 577.

7. FReybuRG, R. H. and PEeT, M. M. (1937). - The effect on the kidney of bilateral splanchnicectomy in patients with hypertension. Jl. Clin. Invest., Vol. XVI, p. 49.

8. PAge, I. H. and HEUER, G. J. (1937).-Treatment of essential and malignant hypertension by section of the anterior nerve roots. Arch. Int. Med., Vol. LIX, p. 245.

9. Goldblatt, H., Lynch, J., Hanzal, R. F. and Summerville, W. W. (1934). - Studies on experimental hypertension. 1. The production of persistent elevation of systolic blood pressure by means of renal ischaemia. Jl. Exper. Med., Vol. LIX, p. 347.

10. WoOD, J. E. and CASH, J. R. (1936).-Experimental hypertension observations on sustained elevation of systolic and diastolic pressure in dog. Jl. Clin. Invest., Vol. XV, p. 543.

11. Grimson, K. S., Wilson, H. and Phemister, D. B. (1937).-The early and remote effects of total and partial paravertebral sympathectomy on blood pressure. Ann. Surg., Vol. CVI, p. 101.

12. Goldblatt, H., Gross, J. and Hanzal, R. F. (1937).-Studies on experimental hypertension. 2. The effect of resection of the splanchnic nerves on experimental renal hypertension. Jl. Exp. Med., Vol. LXV, p. 233.

13. RICKER, G. (1927).--Sklerose und Hypertonie der innervierten Arterien. Julius Springer, Berlin.

\title{
THE TABETIC PUPIL
}

BY

\author{
Percival W. Leathart, M.B., B.C.(Cantab.) \\ SENIOR SURGEON, LIVERPOOL EYE AND EAR INFIRMARY, \\ AND MEMBER OF ITS OTO-LARYNGOLOGICAL CLINIC
}

THE normal movements of the iris are brought about by reflex stimulation of the three intrinsic ocular muscies.

1. The dilator iridis.

2. The constrictor iridis.

3. The ciliary muscle.

Associated with the action of each muscle is a primary nervous reflex.

1. The dilator reflex.

2. The constrictor reflex.

3. The accommodation reflex.

In order to appreciate the significance of abnormalities in the movements and size of the pupil in disease, it is essential to have a clear idea of the anatomy of the various pathways on both the afferent and efferent sides. 
The dilator reflex-mediated by sympathetic fibres.-Although experimental proof is lacking, it is generally conceded that the afferent fibres of the dilator reflex leave the retina in company with the visual fibres in the optic nerve.

After a partial decussation in the chiasma they reach the optic tract, and enter the midbrain through the brachium of the superior colliculus. In this region they gain access to some extrapyramidal tract, possibly the tectospinal, and in it pass through the midbrain, pons, medulla and cervical spinal cord to reach cells in the lateral horns of $\mathrm{C}_{8}-\mathrm{D}_{3}$-the so-called cilio-spinal centre.

In this region of the spinal cord there is said to be a decussation of the afferent dilator fibres which renders the consensual reaction possible.

Here the afferent pathway ends and the efferent begins. The efferent fibres, taking origin from the cells in the lateral horns of $\mathrm{C}_{8}-\mathrm{D}_{3}$, leave the spinal cord as preganglionic fibres via the anterior roots and anterior primary divisions of these segments and enter the cervical sympathetic chain by the white rami connecting it to these segments, and pass up the cervical sympathetic to the superior cervical ganglion where a final relay takes place. The efferent pathway is continued by post-ganglionic fibres through the carotid and cavernous plexuses to the ophthalmic artery and pass with it through the optic foramen. In the orbit they come into contact with but do not relay in the ciliary ganglion and pass into the bulbous oculi with the short ciliary nerves to reach the dilator iridis.

The stimulus which activates the dilator reflex is a decrease in the intensity of light. A lesion in the afferent or efferent limb of this reflex will paralyse the dilator muscle and thus allow the unopposed constrictor to contract the pupil. Such a pupil is seen after cervical sympathectomy, where the homolateral pupil is smaller than its fellow and contracts still more to light.

The constrictor reflex-mediated by parasympathetic fibres.The afferent fibres leave the retina in the optic nerve and have been traced through it and the optic tract. After reaching the lateral aspect of the midbrain the fibres leave the optic tract and enter the midbrain through the brachium of the superior colliculus. They do not, however, enter this structure but gain access to cells in the pretectal region where the first relay takes place. From these cells fresh fibres arise which pass to the Edinger-Westphall nucleus of both sides - the para-sympathetic nuclei of the third nerve nucleus.

This anatomical detail explains the consensual light reflex. Here the afferent limb ends and the efferent begins in cells analogous to those in the lateral horns of $\mathrm{C}_{8}-\mathrm{D}_{3}$. The efferent fibres enter the orbit in the trunk of the third nerve and, after a short journey 
in the inferior division, leave the nerve to the inferior oblique muscle as the short root of the ciliary ganglion. After a final relay in this ganglion they leave it in the short ciliary nerves, enter the bulbous oculi and end in the constrictor iridis.

The stimulus which activates the constrictor reflex is an increase in the intensity of light. A lesion of its afferent or efferent limb or of its centre (Edinger-Westphall nucleus) in the mid-brain, causes paralysis of the constrictor iridis and therefore a dilated pupil. In addition since such a lesion destroys the light reflex contraction to light cannot be obtained.

The accommodation-constrictor reflex. - When an object is brought close to the eyes three separate reactions take place simultaneously.

1. The eyes converge,

2. The ciliary muscle contracts,

3 . The pupils constrict.

The object of these phenomena is to shut off peripheral rays, and to increase the curvature of the lens, so that an image which would otherwise be blurred or seen double is perceived clearly as a single image.

By means of suitable lenses (prisms) it is possible to prevent convergence without interfering with accommodation or constriction. Moreover cases are seen from time to time suffering from the ocular type of progressive muscular atrophy in which, in spite of paralysis of both internal recti, rendering convergence impossible, yet near vision is accompanied by accommodation and pupillary constriction. It is clear therefore that accommodation and pupillary constriction can take place in the absence of convergence and that in such cases the stimulus for this reaction is a blurred image seen in the occipital striate cortex.

Conversely, in certain cases of blindness, voluntary convergence produces pupillary constriction. Since, in this case, the object is not seen, a blurred image cannot be the stimulus. Clearly afferent proprioceptive impulses caused by contraction of the two internal recti are effective in producing pupillary constriction.

The accommodation-constriction reflex is therefore produced by two different stimuli :

1. A blurred image,

2. Proprioceptive impulses arising from the contraction of the two internal recti.

Normally the two stimuli act in conjunction with one another but should one be in abeyance the other can produce the desired result.

The afferent portion of the blurred image reflex is complex and has not been thoroughly worked out, so that the following suggested pathway is necessarily tentative.

Starting from the retina it passes along the visual fibres of the optic nerve and tract to the lateral geniculate body and from here 
to the striate cortex by the optic radiations where the blurred image is perceived. This initiates an impulse to area 19 (Brodmann), stimulation of which causes constriction of the pupil. From here fibres pass in some cortical association pathway and reach the internal capsule down which they pass to gain that portion of the motor oculi nucleus in the mid-brain known as the nucleus of Perlia. This nucleus like that of Edinger-Westphall is parasympathetic in function. From cells in the nucleus of Perlia the efferent pathway reaches the ciliary muscle and the constrictor iridis by parasympathetic fibres. These fibres unlike those of the efferent constrictor reflex from Edinger-Westphall's nucleus do not pass through the ciliary ganglion. This fact is proved by the experiments of Foester Gagel and Mahoney, who find that ablation of the ciliary ganglion abolishes the light reflex but not the pupillary constriction to convergence. It follows therefore that the final common pathway for the accommodation-constrictor reflex is discrete from that of the constrictor reflex. The details of this pathway have not yet been worked out.

The afferent limb of the convergence reflex passes from the two internal recti through the trunk of the motor-oculi nerve to the nucleus of Perlia.

The efferent limb, or final common path, is common to it and that of the blurred image, and discrete from that of the efferent constrictor reflex pathway.

In addition to the reflexes already described there are others activated by emotion, e.g., anger, fear, pain etc., which arise at higher physiological levels than the mid-brain, viz. cortical, thalamic and hypothalamic. The pathways by which contact is made between these on the one hand and the dilator constrictor and accommodation reflexes on the other have not been carefully worked out.

Since intracranial lesions affecting these reflexes above the midbrain do not permanently interfere with the reflex movements of the iris no further mention of them will be made in the discussion which follows.

The tabetic pupil.-In neuro-syphilis abnormal movements of the iris are frequently seen, especially in tabes dorsalis. The typical pupil found in this disease was first described by Argyll Robertson and has been known by his name ever since. Such a pupil has the following characteristics :

1. It is small.

2. It does not dilate in the dark.

3 . It does not contract in the light.

4. It contracts normally to convergence and accommodation.

Argyll Robertson did not explain how a pupil with the above characteristics was produced, and although since his day many 
attempts have been made to explain it, none so far has been completely convincing. Moreover the Argyll Robertson pupil has not yet been produced experimentally. (Fulton 1938.)

On the assumption that the description of the anatomy of the primary ocular reflexes given above is in the main correct, it is possible, in fact easy, to postulate a lesion which will cause a pupil with all the characteristics of the tabetic pupil. This hypothetical lesion must damage the dilator reflex in order to cause a small pupil which does not dilate in the dark. It must also paralyse the constrictor reflex to produce a pupil which will not contract to light. In addition it must leave the accommodation convergence reflex uninjured.

Let us place this lesion in the brachium of the superior colliculus. In this position the afferent fibres of both the constrictor and dilator reflexes are in close proximity and would inevitably be damaged. The afferent fibres of the accommodation reflex, however, have previously left the optic tract to enter the lateral geniculate body and are therefore left intact.

A lesion in the brachium of the superior colliculus therefore will produce a small pupil which will not dilate in the dark or contract in the light, but will react to accommodation and convergence. In short a pupil with all the attributes of the Argyll Robertson pupil. This hypothetical lesion must of course damage the brachia of both sides. There is both pathological and neurological evidence in favour of this contention.

\section{Pathological Evidence}

We know that the syphilitic poison has a predilection for afferent sensory fibres and that in neuro-syphilis their destruction in the posterior roots is responsible for the loss of both knee and ankle reflexes.

The reason why the syphilitic poison selects afferent fibres in this situation is not clear. We should remember, however, that the fibres in question are small myelinated fibres, and in their passage from the posterior root ganglion to the cord are bathed in the cerebro-spinal fluid in the arachnoid space. In neurosyphilis this fluid always contains the syphilitic poison and it may well be that the small myelinated fibres of the posterior roots are more vulnerable to the poison in this situation than the large myelinated fibres of the anterior roots.

The position with regard to the afferent fibres of the ocular reflexes as they enter the mid-brain is exactly similar. Those in the brachium of the superior colliculus subserving the dilator and constrictor reflexes are small myelinated fibres, those entering the lateral geniculate body activating the accommodation-convergence reflex being the visual fibres themselves are large myelinated fibres -both sets of fibres are surrounded by infected cerebro-spinal fluid - the former are destroyed, the latter remain uninjured. 
The above evidence is enhanced by the fact that it brings the pathology of the tabetic pupil in line with that of the loss of knee and ankle reflexes so commonly seen in tabes.

\section{Neurological Evidence}

In order to produce tabetic pupils by means of a mid-brain lesion we must postulate one which injures both Edinger-Westphall nuclei in order to paralyse the constrictor reflexes. It must also damage both sympathetic tracts after they have left the brachia of the superior colliculi in order to produce small pupils. In addition this lesion must not only leave the nucleus of Perlia intact so that constriction on accommodation can be obtained but also avoid all the other nuclei and tracts of this crowded region.

It is true that a pupil inactive to light but active to accommodation is sometimes seen in mid-brain tumours and in the ocular type of progressive muscular atrophy. In these conditions, however, this pupil is almost invariably unilateral at first, usually large, and accompanied by other physical signs not present in tabes. Neurologically it is well nigh impossible to conceive of a mid-brain lesion capable of producing tabetic pupils without causing additional physical signs not present in tabes.

It is extremely improbable, therefore, that a mid-brain lesion is responsible for the tabetic pupil; the balance of evidence is strongly in favour of a peripheral origin, the site of which is the brachia of the superior colliculi.

\section{Summary}

1. The anatomy of the three primary ocular reflexes is described in detail.

2. The characteristics of the tabetic pupil are enumerated.

3. It is shown how it is almost impossible for a mid-brain lesion to produce pupils with the characteristics of a tabetic pupil without at the same time causing other physical signs.

4. It is pointed out that a hypothetical lesion in the brachium of the superior colliculus of both sides produces pupils with all the characteristics of the tabetic pupil.

5. It is postulated that such lesions are present in tabetics and are the cause of the Argyll Robertson pupil.

6. A possible explanation of the fact that syphilitic poison destroys afferent fibres of posterior spinal roots and of the brachia of the superior colliculi is given.

7. It is postulated that such lesions are present in tabes in the posterior roots and in the brachia of the superior colliculi and are the cause of absent knee and ankle reflexes and of the Argyll Robertson pupils respectively. 